\title{
UNIQUENESS OF POSITIVE SOLUTIONS OF NONLINEAR SECOND-ORDER EQUATIONS
}

\author{
ROBERT DALMASSO
}

(Communicated by Hal L. Smith)

\begin{abstract}
In this paper we study the uniqueness question of positive solutions of the two-point boundary value problem: $u^{\prime \prime}(t)+f(|t|, u(t))=0,-R<$ $t<R, u( \pm R)=0$ where $R>0$ is fixed and $f:[0, R] \times[0, \infty) \rightarrow \mathbb{R}$ is in $C^{1}([0, R] \times[0, \infty))$. A uniqueness result is proved when $f$ satisfies some appropriate conditions. Some examples illustrating our theorem are also given.
\end{abstract}

\section{INTRODUCTION}

In this paper we study the question of uniqueness for solutions $u \in$ $C^{2}([-R, R])$ of the two-point boundary value problem

$$
\begin{cases}u^{\prime \prime}(t)+f(|t|, u(t))=0, & -R<t<R, \\ u( \pm R)=0, & -R<t<R, \\ u(t)>0, & \end{cases}
$$

where $R>0$ is fixed and the function $f:[0, R] \times[0, \infty) \rightarrow \mathbb{R}$ is in $C^{1}([0, R] \times$ $[0, \infty))$ and satisfies the following hypotheses:

$\left(\mathrm{H}_{1}\right) \quad u f_{u}(t, u)>f(t, u)$ for $(t, u) \in[0, R] \times(0, \infty)$.

$\left(\mathrm{H}_{2}\right) \quad f_{t}(t, u) \leq 0$ for $(t, u) \in[0, R] \times[0, \infty)$.

$\left(\mathrm{H}_{3}\right)$ There exists $u>0$ such that $f(R, u) \geq 0$.

Conditions for the existence of solutions of (1.1) were studied by many authors: see for instance De Figueiredo, Lions and Nussbaum [2], Granas, Guenther and Lee [4], Lions [8], Rabinowitz [10] and the references therein.

The uniqueness question for problem (1.1) was studied in the case where $f(|t|, u)$ is nonnegative, nondecreasing in $u$ and concave in $u$ in the following generalized sense: for $u>0$ and arbitrary $\tau \in(0,1), f(|t|, \tau u)-\tau f(|t|, u)>0$ for $t \in(-R, R)$ (see for instance Krasnoselskii [6]). A uniqueness result was also established by $\mathrm{Ni}$ and Nussbaum [9] for a different nonlinearity. When $f(|t|, u)$ is nonincreasing in $u$, uniqueness is easily obtained: see Granas, Guenther and Lee [4]. Recently, in the particular case where $f(|t|, u)=$ $p(|t|) f(u)$, several uniqueness results have been established in [1] under weaker regularity assumptions.

Received by the editors April 29, 1994.

1991 Mathematics Subject Classification. Primary 34B15.

Key words and phrases. Two-point boundary value problem, positive solutions, uniqueness. 
We shall prove the following theorem.

Theorem 1. Let $f \in C^{1}([0, R] \times[0, \infty))$ satisfy $\left(\mathrm{H}_{1}\right)-\left(\mathrm{H}_{3}\right)$. Then problem (1.1) has at most one solution in $C^{2}([-R, R])$.

Remark 1. It follows from a result of Gidas, Ni and Nirenberg [3] that under the assumption $\left(\mathrm{H}_{2}\right)$ any solution $u \in C^{2}([-R, R])$ of problem (1.1) is necessarily symmetric about the origin and such that $u^{\prime}<0$ on $(0, R)$. Therefore solutions of (1.1) can be treated as positive solutions of

$$
\left\{\begin{array}{l}
u^{\prime \prime}(t)+f(t, u(t))=0, \quad 0 \leq t<R, \\
u(R)=u^{\prime}(0)=0 .
\end{array}\right.
$$

The key ingredient is the use of the function

$$
\varphi(t, \alpha)=\frac{\partial u}{\partial \alpha}(t, \alpha)
$$

where $u$ is considered as a function of both $t$ and the parameter $\alpha=u(0)$. The main point is to show that $\varphi$ changes sign only once in $[0, R]$ and that $\varphi(R, u(0))<0$.

The function $\varphi$ has been used in cases where $f$ in (1.2) also depends on $u^{\prime}$ and even when $f$ is singular at $t=0$. For instance Kwong [7] established uniqueness for the following boundary value problems:

$$
\begin{aligned}
& u^{\prime \prime}(t)+\frac{m}{t} u^{\prime}(t)+u(t)^{p}-u(t)=0, \quad u(t)>0,0 \leq a<t<b \leq \infty, \\
& u^{\prime}(a)=0, \\
& u(b)=0 \text { if } b<\infty
\end{aligned}
$$

or

$$
\lim _{t \rightarrow \infty} u(t)=0 \quad \text { if } b=\infty
$$

with $m \geq 0, p>1$ and $p<(m+3) /(m-1)$ if $a=0$ and $m>1$.

In Section 2 we introduce an initial value problem for the differential equation in (1.2) and we state our main result-Theorem 2-from which we derive Theorem 1. Section 3 is devoted to the proof of Theorem 2: some crucial lemmas are needed. Finally some examples to which our theorem applies are given in Section 4.

\section{MAIN ReSUlts}

Let $f \in C^{1}([0, R] \times[0, \infty))$. If we set

$$
f(t, u)=f(R, u) \text { for } t>R
$$

and

$$
f(t, u)=f(t, 0)+u f_{u}(t, 0) \text { for } u<0,
$$

then we can assume that $f \in C([0, \infty) \times(-\infty, \infty))$ and that $f$ is continuously differentiable in $u$. Moreover if $\left(\mathrm{H}_{2}\right)$ is verified we can assume that $f$ is nonincreasing in $t \geq 0$ when $u \geq 0$.

Now we introduce the initial value problem

$$
\begin{gathered}
u^{\prime \prime}(t)+f(t, u(t))=0, \quad t>0, \\
u(0)=\alpha, \quad u^{\prime}(0)=0
\end{gathered}
$$


where $\alpha>0$ is a parameter. The unique solution of this problem will be denoted by $u(\cdot, \alpha)$ or $u . u(\cdot, \alpha)$ is defined on a maximum interval $\left[0, T_{\alpha}\right)$ $\left(T_{\alpha}=\infty\right.$ possibly). We define $t(\alpha)$ by

$$
t(\alpha)=\sup \left\{t \in\left[0, T_{\alpha}\right) ; u(s, \alpha)>0 \text { for } s \in[0, t)\right\} .
$$

If $u(t, \alpha)>0$ for all $t \in\left[0, T_{\alpha}\right)$, then $t(\alpha)=T_{\alpha}$ and if $u(\cdot, \alpha)$ has a finite zero in $\left[0, T_{\alpha}\right), t(\alpha)$ denotes the first zero of $u(\cdot, \alpha)$ in $\left[0, T_{\alpha}\right)$.

Remark 2. When $t(\alpha)<T_{\alpha}$ we have $u(t(\alpha), \alpha)=0$. Under the assumption $\left(\mathrm{H}_{2}\right)$ the solution $u(\cdot, \alpha)$ of problem $(2.1),(2.2)$ is such that $u^{\prime}(t, \alpha)<0$ for $t \in(0, t(\alpha))$ : as before this follows from a result of Gidas, Ni and Nirenberg [3].

We introduce the following definition.

Definition. The solution $u(\cdot, \alpha)$ of problem $(2.1),(2.2)$ is said to be admissible if

(i) $t(\alpha) \leq R$;

(ii) $u^{\prime}(t, \alpha) \leq 0$ for $t \in[0, t(\alpha))$;

(iii) $f(0, \alpha)>0$.

Remark 3. Let $u(\cdot, \alpha)$ be an admissible solution of problem (2.1), (2.2). (ii) implies that $u(\cdot, \alpha)$ is bounded on $[0, t(\alpha))$. Therefore $t(\alpha)<T_{\alpha}$ and hence $u(t(\alpha), \alpha)=0$.

Now we can state our main result.

Theorem 2. Let $f \in C^{1}([0, R] \times[0, \infty))$ satisfy $\left(\mathrm{H}_{1}\right)-\left(\mathrm{H}_{3}\right)$. If the solution $u\left(\cdot, \alpha_{0}\right)$ of problem (2.1), (2.2) is admissible for some $\alpha_{0}>0$, then we have:

(i) $u(\cdot, \alpha)$ is admissible for all $\alpha \in\left[\alpha_{0}, \infty\right)$;

(ii) $t(\alpha)$ is a strictly decreasing function of $\alpha \in\left[\alpha_{0}, \infty\right)$.

The proof of Theorem 1 is now easy. Let $u \in C^{2}([-R, R])$ be a solution of problem (1.1). By Remark $1 u$ can be treated as a positive solution of problem (1.2). Therefore $u$ is the solution of problem (2.1), (2.2) such that $\alpha=u(0)$ and $t(\alpha)=R<T_{\alpha}$. We first show that $u$ is admissible. By Remark 1 again it is enough to show that $f(0, u(0))>0$. If not, $\left(\mathrm{H}_{2}\right)$ implies that $f(t, u(0)) \leq 0$ for all $t \in[0, R]$. Then by $\left(\mathrm{H}_{1}\right)$ we deduce that $f(t, u)<0$ for $t \in[0, R]$ and $u \in(0, u(0))$. Thus $u^{\prime \prime}>0$ on $(0, R)$ from which we get $u^{\prime}>0$ on $(0, R)$ and we reach a contradiction. Now let $v \in C^{2}([-R, R])$ be another solution of problem $(1.1)$ and assume for instance that $u(0)<v(0)$. Since $t(u(0))=t(v(0))=R$, we get a contradiction with (ii) in Theorem 2.

\section{Proof of Theorem 2}

We first state and prove two lemmas ( 1 and 4$)$ which are needed in the proof of Theorem 2.

Lemma 1. Assume $\left(\mathrm{H}_{1}\right)$ and $\left(\mathrm{H}_{2}\right)$. Let $u(\cdot, \alpha)$ be an admissible solution of problem (2.1), (2.2). Then $\varphi=\varphi(\cdot, \alpha)=\frac{\partial u}{\partial \alpha}(\cdot, \alpha)$ is such that $\varphi(t(\alpha), \alpha)<0$.

We shall need two lemmas. 
Lemma 2. Assume $\left(\mathrm{H}_{1}\right)$ and $\left(\mathrm{H}_{2}\right)$. Let $u(\cdot, \alpha)$ be an admissible solution of problem (2.1), (2.2). Then

(i) $t(\alpha)<T_{\alpha}, u(t(\alpha), \alpha)=0$ and $u^{\prime}(t, \alpha)<0$ for $t \in(0, t(\alpha))$;

(ii) $\left(u^{\prime} / u\right)^{\prime}<0$ on $(0, t(\alpha))$.

Proof. (i) is given by Remark 3 and Remark 2. We now prove (ii). We have

$$
u(t)^{2}\left(u^{\prime} / u\right)^{\prime}(t)=u^{\prime \prime}(t) u(t)-u^{\prime}(t)^{2}=-\left(u(t) f(t, u(t))+u^{\prime}(t)^{2}\right)
$$

for $t \in(0, t(\alpha))$. Denoting by $\psi(t)$ the right-hand side in (3.1) we get

$$
\psi^{\prime}(t)=-\left\{u(t) f_{t}(t, u(t))+\left(u(t) f_{u}(t, u(t))-f(t, u(t))\right) u^{\prime}(t)\right\}
$$

for $t \in(0, t(\alpha))$. Using (i), $\left(\mathrm{H}_{1}\right)$ and $\left(\mathrm{H}_{2}\right)$ we obtain $\psi^{\prime}(t)>0$ for $t \in$ $(0, t(\alpha))$ and hence $\psi(t)<\psi(t(\alpha))=-u^{\prime}(t(\alpha), \alpha)^{2} \leq 0$ for $t \in(0, t(\alpha))$ and the lemma follows.

Lemma 3. Assume $\left(\mathrm{H}_{1}\right)$ and $\left(\mathrm{H}_{2}\right)$. Let $u(\cdot, \alpha)$ be an admissible solution of problem (2.1), (2.2). Then $\lim _{t \rightarrow t(\alpha)^{-}}\left(u^{\prime} / u\right)(t)=-\infty$.

Proof. The lemma is clear when $u^{\prime}(t(\alpha), \alpha)<0$. Now assume that $u^{\prime}(t(\alpha), \alpha)$ $=0$. Then necessarily $f(t(\alpha), 0)<0$. Indeed $\left(\mathrm{H}_{1}\right)$ implies that $f(t, 0) \leq 0$ for $t \in[0, t(\alpha)]$. Thus, if $f(t(\alpha), 0)=0$, we deduce from $\left(\mathrm{H}_{2}\right)$ that $f(t, 0)=$ 0 for $t \in[0, t(\alpha)]$. Therefore the uniqueness theorem for the initial value problem for ordinary differential equations implies that $u=0$ on $[0, t(\alpha)]$ and we reach a contradiction. Now we have $u^{\prime \prime}(t(\alpha), \alpha)=-f(t(\alpha), 0)>0$ and the lemma follows.

Proof of Lemma 1. For $\beta \geq 0$ we define the function $u_{\beta}=u+\beta u^{\prime}$. By Lemmas 2 and 3 , for any $\beta>0$ there exists a unique $r(\beta) \in(0, t(\alpha))$ such that

$$
u_{\beta}>0 \text { on }[0, r(\beta)) \quad \text { and } \quad u_{\beta}<0 \quad \text { on }(r(\beta), t(\alpha)) .
$$

Moreover $r(\beta)$ is a strictly decreasing continuous function of $\beta$ satisfying

$$
r(0)=t(\alpha) \quad \text { and } \quad r(\beta) \rightarrow 0 \quad \text { as } \beta \rightarrow \infty .
$$

Differentiating (2.1) with respect to $\alpha$ and using (2.2), we obtain

$$
\begin{gathered}
\varphi^{\prime \prime}(t)+f_{u}(t, u(t)) \varphi(t)=0 \quad \text { for } t \in[0, t(\alpha)), \\
\varphi(0)=1, \quad \varphi^{\prime}(0)=0 .
\end{gathered}
$$

We can write

$$
\begin{aligned}
u^{\prime}(t(\alpha)) \varphi(t(\alpha)) & =\int_{0}^{t(\alpha)}\left\{u^{\prime \prime}(t) \varphi(t)-\varphi^{\prime \prime}(t) u(t)\right\} d t \\
& =\int_{0}^{t(\alpha)} \varphi(t)\left\{u(t) f_{u}(t, u(t))-f(t, u(t))\right\} d t .
\end{aligned}
$$

Assume that $\varphi(t)>0$ for $t \in[0, t(\alpha))$. Then, by $\left(\mathrm{H}_{1}\right)$ the right-hand side is positive. Since the left-hand side is nonpositive, we obtain a contradiction. Thus we can define $t_{1}$ to be the first zero of $\varphi$ in $(0, t(\alpha))$. We shall show that $t_{1}$ is the unique zero of $\varphi$ in $(0, t(\alpha)]$. Suppose the contrary. Then we 
denote by $t_{2}$ the first zero of $\varphi$ in $\left(t_{1}, t(\alpha)\right.$ ]. Using (3.2) we can choose $\beta>0$ such that $r(\beta) \leq t_{1}$. Then we write

$$
\begin{aligned}
-\varphi^{\prime} & \left(t_{2}\right) u_{\beta}\left(t_{2}\right)+\varphi^{\prime}\left(t_{1}\right) u_{\beta}\left(t_{1}\right) \\
& =\int_{t_{1}}^{t_{2}}\left\{u_{\beta}^{\prime \prime}(t) \varphi(t)-\varphi^{\prime \prime}(t) u_{\beta}(t)\right\} d t \\
& =\int_{t_{1}}^{t_{2}} \varphi(t)\left\{u(t) f_{u}(t, u(t))-f(t, u(t))-\beta f_{t}(t, u(t))\right\} d t .
\end{aligned}
$$

By $\left(\mathrm{H}_{1}\right),\left(\mathrm{H}_{2}\right)$ and the fact that $\varphi<0$ on $\left(t_{1}, t_{2}\right)$ the right-hand side is negative. Since the left-hand side is positive, we obtain a contradiction. Thus $t_{1}$ is the unique zero of $\varphi$ in $(0, t(\alpha)]$ and the lemma follows.

Lemma 4. Assume $\left(\mathrm{H}_{1}\right)$ and $\left(\mathrm{H}_{2}\right)$. Let $u(\cdot, \alpha)$ be an admissible solution of problem (2.1), (2.2). Then there exists $\eta>0$ such that $u(\cdot, \gamma)$ is admissible for all $\gamma \in[\alpha, \alpha+\eta)$.

Proof. Let $\gamma>\alpha$ with $\gamma-\alpha$ sufficiently small. By Remark 3 we have $t(\alpha)<T_{\alpha}$. Since $\alpha \rightarrow T_{\alpha}$ is a lower semicontinuous function (see Hartman [5] Theorem 2.1 p. 94), $t(\alpha)<T_{\gamma}$. Now we have

$$
u(t(\alpha), \gamma)=(\gamma-\alpha) \varphi(t(\alpha), \alpha)+o(\gamma-\alpha) .
$$

By Lemma $1 \varphi(t(\alpha), \alpha)<0$; then $u(t(\alpha), \gamma)<0$. By the intermediate value theorem we deduce that $t(\gamma)<t(\alpha)$. Using Remark 2 we have $u^{\prime}(t, \gamma)<0$ on $(0, t(\gamma))$. Since $\gamma>\alpha$, we have $f(0, \gamma)>f(0, \alpha)$ by $\left(\mathrm{H}_{1}\right)$. The lemma is proved.

Let $F(t, u)=\int_{0}^{u} f(t, s) d s$. We shall also need the following lemma.

Lemma 5. Assume $\left(\mathrm{H}_{1}\right)-\left(\mathrm{H}_{3}\right)$. Then:

(i) For any $t \in[0, R]$ there exists $\beta(t) \in[0, \infty)$ such that $f(t, u)<0$ for $u \in(0, \beta(t))$ and $f(t, u)>0$ for $u \in(\beta(t), \infty)$. Moreover $\beta$ is $a$ nondecreasing function.

(ii) There exists $u>0$ such that $F(R, u) \geq 0$.

(iii) For any $t \in[0, R]$ there exists $\gamma(t) \in[0, \infty)$ such that $F(t, u)<$ 0 for $u \in(0, \gamma(t))$ and $F(t, u)>0$ for $u \in(\gamma(t), \infty)$. Moreover $\gamma$ is a nondecreasing function.

Proof. (i) is clear. We now prove (ii). Let $v>\beta(R)$ be fixed. Using (i) and $\left(\mathrm{H}_{1}\right)$ we have

$$
f(R, u)>u f(R, v) / v>0 \text { for } u>v .
$$

Therefore for $u>v$ we can write

$$
\begin{aligned}
F(R, u) & =\int_{0}^{v} f(R, s) d s+\int_{v}^{u} f(R, s) d s \\
& >\int_{0}^{v} f(R, s) d s+\left(u^{2}-v^{2}\right) f(R, v) / 2 v
\end{aligned}
$$

and (ii) follows. Now from $\left(\mathrm{H}_{1}\right)$ (resp. $\left(\mathrm{H}_{2}\right)$ ) we deduce that $u f(t, u)>$ $2 F(t, u)$ for $(t, u) \in[0, R] \times(0, \infty)$ (resp. $F_{t}(t, u) \leq 0$ for $(t, u) \in[0, R] \times$ $[0, \infty))$. Then (iii) is immediate and the lemma is proved. 
We conclude this section with the proof of Theorem 2 .

(i) Suppose that there exists $\alpha \in\left(\alpha_{0}, \infty\right)$ such that $u(\cdot, \alpha)$ is not admissible and define

$$
\alpha_{1}=\inf \left\{\alpha \in\left(\alpha_{0}, \infty\right) ; u(\cdot, \alpha) \text { is not admissible }\right\}<\infty .
$$

By Lemma $4 u\left(\cdot, \alpha_{1}\right)$ is not admissible. Since $\alpha_{1}>\alpha_{0},\left(\mathrm{H}_{1}\right)$ implies that $f\left(0, \alpha_{1}\right)>f\left(0, \alpha_{0}\right)>0$. Therefore we have two cases to consider.

Case 1: $t\left(\alpha_{1}\right)>R$. As before $T_{\alpha}>R$ for $\left|\alpha-\alpha_{1}\right|$ sufficiently small. The continuity of $u(\cdot, \alpha)$ with respect to the initial value $\alpha$ implies that there exists $\eta>0$ such that $u(t, \alpha)>0$ for $t \in[0, R]$ and $\alpha \in\left(\alpha_{1}-\eta, \alpha_{1}+\eta\right)$. Thus we obtain a contradiction with the fact that $u(\cdot, \alpha)$ is admissible for all $\alpha \in\left[\alpha_{0}, \alpha_{1}\right)$.

Case 2: $t\left(\alpha_{1}\right) \leq R$ and there exists $t_{0} \in\left(0, t\left(\alpha_{1}\right)\right)$ such that $u^{\prime}\left(t_{0}, \alpha_{1}\right)>0$. By Remark $2 t\left(\alpha_{1}\right)=T_{\alpha_{1}}$. Then there exists $s_{n} \in\left(0, t\left(\alpha_{1}\right)\right)$ such that $s_{n} \rightarrow t\left(\alpha_{1}\right)$ and $u\left(s_{n}, \alpha_{1}\right) \rightarrow \infty$ as $n \rightarrow \infty$. Using $\left(\mathrm{H}_{2}\right)$ we have

$$
\frac{d}{d t}\left(u^{\prime}\left(t, \alpha_{1}\right)^{2} / 2+F\left(t, u\left(t, \alpha_{1}\right)\right)\right)=F_{t}\left(t, u\left(t, \alpha_{1}\right)\right) \leq 0 \text { for } t \in\left[0, t\left(\alpha_{1}\right)\right) .
$$

Since $f\left(0, \alpha_{1}\right)>0$, there exists $\eta>0$ such that $u^{\prime}\left(t, \alpha_{1}\right)<0$ for $t \in$ $(0, \eta)$. From $u^{\prime}\left(t_{0}, \alpha_{1}\right)>0$ we deduce that there exists $t_{1} \in\left(0, t_{0}\right)$ such that $u^{\prime}\left(t_{1}, \alpha_{1}\right)=0$ and $u^{\prime \prime}\left(t_{1}, \alpha_{1}\right) \geq 0$. Therefore $f\left(t_{1}, u\left(t_{1}, \alpha_{1}\right)\right) \leq 0$ from which we get, using (i) in Lemma $5, F\left(t_{1}, u\left(t_{1}, \alpha_{1}\right)\right)<0$. Thus $u^{\prime}\left(t_{1}, \alpha_{1}\right)^{2} / 2+$ $F\left(t_{1}, u\left(t_{1}, \alpha_{1}\right)\right)<0$. By virtue of (iii) in Lemma 5 we can choose $n$ such that $s_{n}>t_{1}$ and $F\left(R, u\left(s_{n}, \alpha_{1}\right)\right)>0$. Then using $\left(\mathrm{H}_{2}\right)$ we have $u^{\prime}\left(s_{n}, \alpha_{1}\right)^{2} / 2+$ $F\left(s_{n}, u\left(s_{n}, \alpha_{1}\right)\right) \geq F\left(R, u\left(s_{n}, \alpha_{1}\right)\right)>0$ and we get a contradiction with (3.5).

(ii) Let $\alpha \in\left[\alpha_{0}, \infty\right)$ and $\gamma>\alpha$ with $\gamma-\alpha$ sufficiently small. Using (i) we show that $t(\gamma)<t(\alpha)$ in the same way as in the proof of Lemma 4.

The proof of the theorem is complete.

Remark 4. Let $f \in C^{1}([0, R] \times[0, \infty))$ be such that $f(t, u) \geq 0$ for $(t, u) \in$ $[0, R] \times[0, \infty)$. Assume $\left(\mathrm{H}_{2}\right)$ and

$\left(\mathrm{H}_{1}\right)^{\prime} \quad u f_{u}(t, u)>f(t, u)$ for $(t, u) \in[0, R) \times(0, \infty)$.

Then Theorems 1 and 2 still hold. We indicate below the slight modifications.

First we note that $\left(\mathrm{H}_{1}\right)^{\prime}$ implies $f(t, 0)=0$ for all $t \in[0, R]$ and that, for all $t \in[0, R), f(t, \cdot)$ is strictly increasing on $[0, \infty)$. Therefore $f(t, u)>0$ for $t \in[0, R)$ and $u>0$. Thus $f(0, u(0))>0$ in the proof of Theorem 1 . In Lemma 3 the case $u^{\prime}(t(\alpha), \alpha)=0$ cannot occur by the uniqueness theorem for the initial value problem for ordinary differential equations. In Lemma 5 (i) and (iii) are still valid for $t \in[0, R)$ with $\beta(t)=\gamma(t)=0$ and (ii) is true for all $u \geq 0$. Actually Lemma 5 is not needed in the present situation. Indeed we note that, in the proof of Theorem 2, Case 2 obviously cannot occur.

\section{EXAMPLES}

4.1. Let $f \in C^{1}([0, \infty), \mathbb{R})$ be such that $u f^{\prime}(u)>f(u)$ for $u>0$. Then Theorem 1 applies. Indeed we note that if $f(u)<0$ for $u>0$, then problem (1.1) has no solutions. We give below some examples for which existence is well known (see [10]). 
(i) $f(u)=\sum_{j=1}^{k} a_{j} u^{p_{j}}$ with $p_{j}>1$ and $a_{j}>0$ for $j=1, \ldots, k$;

(ii) $f(u)=u^{p} /\left(1+u^{s}\right)$ with $p-1>s>0$;

(iii) $f(u)=-u+u^{p}$ with $p>1$.

4.2. Let $f \in C^{1}([0, \infty), \mathbb{R})$ satisfy $f(u) \geq 0$ for $u \geq 0$ and $u f^{\prime}(u)>f(u)$ for $u>0$. Let $a \in C^{1}([0, R])$ be such that $a^{\prime} \leq 0$ on $[0, R], a(t)>0$ for $t \in[0, R)$ and $a(R) \geq 0$. Define

$$
f(t, u)=a(t) f(u) .
$$

Then, by Remark 4, Theorem 1 applies.

4.3. Let $a, b \in C^{1}([0, R])$ be such that $a^{\prime} \geq 0, b^{\prime} \leq 0$ on $[0, R], a(0)>0$ and $b(R)>0$. Define

$$
f(t, u)=-a(t) u+b(t) u^{p} \quad \text { where } p>1 .
$$

Then the existence of a solution of $(1.1)$ is known [10]. Theorem 1 gives uniqueness.

4.4. Let $a, b \in C^{1}([0, R])$ be such that $a^{\prime}, b^{\prime} \leq 0$ on $[0, R]$ and $a(R)$, $b(R)>0$. Define

$$
f(t, u)=\lambda a(t) u+b(t) u^{p}
$$

with $p>1$ and $0 \leq \lambda<\lambda_{1}$ where $\lambda_{1}$ is the smallest eigenvalue of

$$
\left\{\begin{array}{l}
v^{\prime \prime}(t)+\mu a(|t|) v(t)=0, \quad-R<t<R \\
v( \pm R)=0
\end{array}\right.
$$

Then the existence of a solution of (1.1) is well known [10] and Theorem 1 gives uniqueness. If $b(t)>0$ for $t \in[0, R)$ and $b(R) \geq 0$, then, by Remark 4 , Theorem 1 implies uniqueness.

\section{ACKNOWLEDGMENT}

The author is grateful to the referee for his remarks.

\section{REFERENCES}

1. R. Dalmasso, Uniqueness of positive and nonnegative solutions of nonlinear equations, Funkcial. Ekvac. 37 (1994), 461-482.

2. D. De Figueiredo, P.-L. Lions, and R. Nussbaum, A priori estimates and existence of positive solutions of semilinear elliptic equations, J. Math. Pures Appl. (9) 61 (1982), 41-63.

3. B. Gidas, W.-M. Ni, and L. Nirenberg, Symmetry and related properties via the maximum principle, Comm. Math. Phys. 68 (1979), 209-243.

4. A. Granas, R. B. Guenther, and J. W. Lee, Nonlinear boundary value problems for ordinary differential equations, Dissertationes Math. (Rozprawy Mat.) 244 (1985).

5. P. Hartman, Ordinary differential equations, Wiley, New York, 1964.

6. M. Krasnoselskii, Positive solutions of operator equations, Noordhoff, Groningen, 1964.

7. M. K. Kwong, Uniqueness of positive solutions of $\Delta u-u+u^{p}=0$ in $\mathbb{R}^{n}$, Arch. Rational Mech. Anal. 105 (1989), 243-266.

8. P.-L. Lions, On the existence of positive solutions of semilinear elliptic equations, SIAM Rev. 24 (1982), 441-467. 
9. W.-M. Ni and R. Nussbaum, Uniqueness and nonuniqueness for positive radial solutions of $\Delta u+f(u, r)=0$, Comm. Pure Appl. Math 38 (1985), 69-108.

10. P. H. Rabinowitz, Minimax methods in critical point theory with applications to differential equations, CBMS Regional Conf. Ser. in Math., vol. 65, Amer. Math. Soc., Providence, RI, 1986.

Laboratoire LMC-IMAG, Equipe EDP, Tour IRMA, B.P. 53, F-38041 Grenoble Cedex 9, FrANCE

E-mail address: Robert.Dalmasso@imag.fr 